\title{
Influence of deposition parameters on the optical absorption of amorphous silicon thin films
}

\author{
Lukas Terkowski $\odot,{ }^{1}$ Iain W. Martin $\odot,{ }^{2}$ Daniel Axmann, ${ }^{1}$ Malte Behrendsen, ${ }^{1}$ Felix Pein $\odot,{ }^{1}$ Angus Bell, ${ }^{2}$ Roman Schnabel $\odot,{ }^{1}$ \\ Riccardo Bassiri, ${ }^{3}$ Martin M. Fejer, ${ }^{3}$ Jim Hough, ${ }^{2}$ Ashot Markosyan $\odot{ }^{3}$ Sheila Rowan, ${ }^{2}$ and Jessica Steinlechner ${ }^{1,2,4,5}$ \\ ${ }^{1}$ Institut für Laserphysik and Zentrum für Optische Quantentechnologien, Universität Hamburg, \\ Luruper Chaussee 149, 22761 Hamburg, Germany \\ ${ }^{2}$ SUPA, Institute for Gravitational Research, University of Glasgow, Glasgow G12 8QQ, Scotland, United Kingdom \\ ${ }^{3}$ E.L. Ginzton Laboratory, Stanford University, Stanford, California 94305, USA \\ ${ }^{4}$ Maastricht University, P.O. Box 616, 6200 MD Maastricht, The Netherlands \\ ${ }^{5}$ Nikhef, Science Park 105, 1098 XG Amsterdam, The Netherlands
}

(Received 19 February 2020; accepted 24 July 2020; published 26 August 2020)

\begin{abstract}
Amorphous silicon (aSi) is a promising material for application in mirror coatings with low thermal noise in future gravitational-wave detectors. However, the optical absorption of aSi is currently too high to meet the requirements of these instruments. Previously measured absorption values vary significantly for different deposition methods and postdeposition treatments. To investigate the absorption of aSi, we systematically varied key deposition parameters using pulsed laser deposition. Varying the deposition temperature resulted in a spread in mobility gap energy of the aSi; however, no clear correlation of temperature and mobility gap could be observed. Varying the pulse energy and repetition frequency altered the deposition rate of the coating and produced a correlated change in the absorption.
\end{abstract}

DOI: 10.1103/PhysRevResearch.2.033308

\section{INTRODUCTION}

The first detection of gravitational waves in 2015 [1] opened a new window for astronomical observation. Several signals from binary black-hole mergers [2] as well as a binary neutron-star merger [3] were detected in the first two observing runs of the Advanced LIGO [4,5] and Advanced Virgo [6] gravitational-wave detectors. These detectors, based on Michelson interferometers, measure the relative separation between test masses, coated to form highly reflective (HR) mirrors. Gravitational waves induce changes in this separation. Thermal noise associated with the HR mirror coatings will limit the achievable sensitivity of the current detectors when they reach their design sensitivity [5,7]. Thermal noise arises from Brownian motion of the atoms in the coating, and the magnitude increases with the mechanical loss of the coating materials and the temperature of the mirror.

HR coatings are generally made from alternating layers of high- and low-refractive-index materials. Advanced LIGO and Advanced Virgo currently use silica $\left(\mathrm{SiO}_{2}\right)$ as the lowindex material, and titania-doped tantala $\left(\mathrm{TiO}_{2}: \mathrm{Ta}_{2} \mathrm{O}_{5}\right)$ as the high-index material. Both materials possess exceptional optical properties such as low absorption and low scattering [8]. However, the mechanical loss of these coatings is relatively high [9] (dominated by the $\mathrm{TiO}_{2}: \mathrm{Ta}_{2} \mathrm{O}_{5}$ layers), resulting

Published by the American Physical Society under the terms of the Creative Commons Attribution 4.0 International license. Further distribution of this work must maintain attribution to the author(s) and the published article's title, journal citation, and DOI. in a significant level of thermal noise $[10,11]$. The Japanese KAGRA detector-currently under construction [12]-and the proposed European Einstein Telescope (ET) [13] will be operated at cryogenic temperatures to reduce thermal noise. However, there is evidence for an increase in mechanical loss of the currently used coatings at low temperature [14-16] and therefore new coating materials are required. In addition to low mechanical loss, low optical absorption of a few ppm $\left(10^{-6}\right)$ or below is required to maintain the desired cryogenic operating temperature of the mirror.

Amorphous silicon (aSi) shows a very low mechanical loss at cryogenic temperatures $[17,18]$ and appears to be a promising candidate for an alternative high-refractive-index coating material. However, the optical absorption of aSi is high at the currently used detector laser-wavelength of $1064 \mathrm{~nm}$ [19]. Future cryogenic detectors such as ET are likely to use crystalline silicon, which is not transparent at $1064 \mathrm{~nm} \mathrm{[20],}$ for the mirror substrates [13]. Therefore, the wavelength will likely need to be increased. At 1550 or $2000 \mathrm{~nm}$, the optical absorption of aSi has been shown to be significantly lower than at $1064 \mathrm{~nm}$, but still needs to be reduced [19,21,22].

The optical absorption of aSi coatings reduces with postdeposition heat treatment, with a minimum value occurring at $400-500{ }^{\circ} \mathrm{C}[19,21,22]$, and is highly dependent on the deposition process used. Commercial $\mathrm{HR} a \mathrm{Si} / \mathrm{SiO}_{2}$ coatings deposited by ion-beam sputtering (IBS) can have an absorption as high as several thousand ppm at $1550 \mathrm{~nm}$ and at room temperature (RT) [19]. The contribution of the $\mathrm{SiO}_{2}$ layers to the total absorption is negligible. $\mathrm{An} \mathrm{aSi} / \mathrm{SiO}_{2} \mathrm{HR}$ stack deposited using an ion-plating technique has been found to have a significantly lower absorption of $\approx 350 \mathrm{ppm}$ [22]. In this technique, the substrate reaches a temperature of 
approximately $200{ }^{\circ} \mathrm{C}$ during deposition (compared to $\approx 90{ }^{\circ} \mathrm{C}$ for IBS). Even lower absorption-of $7.5 \mathrm{ppm}$ for an $\mathrm{HR}$ aSi $/ \mathrm{SiO}_{2}$ stack-has been obtained for aSi films deposited using a modified IBS method relying on a newly developed type of electron cyclotron resonance (ECR) ion source [21]. This absorption level is still above the requirement for future gravitational-wave detectors and not always reproducible.

To further reduce the absorption and to transfer the process to other deposition systems, a study of the effect of deposition parameters on the coating properties is required. For the ECR-IBS coatings, the absorption has been shown to be positively correlated to the dangling-bond density (i.e., unpaired electrons), which reduced with heat treatment. A correlation of the absorption and the mobility gap energy $E_{\mathrm{g}}$ has also been observed, with the absorption at 1550 and $2000 \mathrm{~nm}$ decreasing towards higher $E_{\mathrm{g}}$. Both of these attributes are a well-known source of optical absorption in aSi [23]. It has been suggested that the low deposition rate and the high ion energy used in this deposition process may be responsible for the low absorption [21]. While it is clear that the deposition technique and postdeposition treatment have an important role in determining the absorption of the film, these effects are not fully understood.

Here, we report on aSi coatings produced using pulsed laser deposition (PLD). We carried out a systematic investigation of the effect of laser-pulse energy, pulse repetition rate, and substrate temperature on $E_{\mathrm{g}}$, and on the real and imaginary parts of the complex refractive index $n=n+i k$.

\section{COATING DEPOSITION AND ANALYSIS METHODS}

In this section we introduce the thin-film deposition method and the characterization techniques used.

\section{A. Pulsed laser deposition}

PLD was used to produce aSi coatings at the University of Hamburg. This method allows for low deposition rates, due to the very small volume of target material vaporized with each laser pulse. In addition, it is a "clean" technique which does not use other atomic species, unlike, e.g., IBS, which relies on energetic ions to sputter the target material.

In PLD, a pulsed laser beam is focused onto a target. In our setup, the laser wavelength was $248 \mathrm{~nm}$ and the pulse width was $20 \mathrm{~ns}$. The focused laser beam was scanned over the target, covering a rectangular area as large and uniform as possible. The scanning speed varied slightly between similar coatings due to different alignment. For higher laser-pulse frequencies, the scanning speed was increased. This scanning routine maintained a large enough distance between successive ablation points, ensuring a uniform ablation of material and preventing dents forming in the target. With each laser pulse, some of the target material is vaporized, expands into a plume, and is deposited onto a substrate mounted about $10 \mathrm{~cm}$ from the target. A rotating-vane velocity filter was used between the target and the substrate to remove slowermoving, larger pieces of material. The substrate temperature was controlled via laser heating of the back surface, with the temperature monitored by an infrared pyrometer [24]. A thorough description of our PLD setup can be found in [25].

The coatings were produced in three series, in which one deposition parameter per series was systematically varied: the laser-pulse repetition frequency $f$, the laser-pulse energy $E$, and the substrate temperature $T$. The depositions were carried out at a pressure of approximately $10^{-5} \mathrm{mbar}$. For the first two series, Corning 7980 fused silica was used as a substrate material. At $1550 \mathrm{~nm}$ the absorption of Corning 7980 silica is negligible compared to the absorption of our coatings. However, at $2000 \mathrm{~nm}$, the absorption of Corning 7980 silica was too high to allow the separation of the coating absorption from the substrate background. Therefore, for the third series we moved to Corning 7979 silica, which has lower absorption at $2000 \mathrm{~nm}$ and allowed measurements to be carried out at this wavelength.

\section{B. Transmission spectra}

Optical transmission spectra in the wavelength region between $200 \mathrm{~nm}$ and $3 \mu \mathrm{m}$ were measured for each coating [26]. The spectra were analyzed with the software tool sCOUT [27].

By utilizing a modified version of the so-called OJL model [28], SCOUT is able to simulate the transmission spectra of our samples. This model describes the distribution of electronic states in amorphous semiconductors to determine the optical absorption due to interband transitions which in turn defines the imaginary part of the electric susceptibility $\chi_{\mathrm{OJL}}$. The modification of the model consists of adding a decay term to reduce the electron state density and therefore the imaginary part of $\chi_{\mathrm{OJL}}$ at high energies down to zero. This makes it possible to calculate the real part of $\chi_{\mathrm{OJL}}$ by exploiting the Kramers-Kronig relations [29]. Furthermore, SCOUT adds an electronic harmonic oscillator to approximate the influence of high-energy interband transitions. Although the energy gap between the eigenstates of the oscillator is outside of the considered region, the real part of its susceptibility $\chi_{\mathrm{HO}}$ is still nonzero at higher wavelengths. Both susceptibilities $\chi_{\mathrm{OJL}}$ and $\chi_{\mathrm{HO}}$ are added and define the dielectric function of the amorphous silicon thin films from which the complex refractive index can be derived.

The transmission spectra can now be calculated and fit to the measurements. It was necessary to include a scattering term to obtain good fits to the measured spectra, as scattering was caused by the microcrystals present in the coatings (see Sec. IID and Fig. 2). The term describes the total scattering effect as it is not relevant to know more details of scattering mechanisms to obtain the relevant parameters of the coatings in this paper.

To begin with, the measured transmission spectra of both types of uncoated fused silica substrates were modeled. The aSi layer on top was then modeled by fitting simulated spectra to the measurements. From the complete model, a number of different parameters for the aSi film can be derived, including the thickness $t$, the mobility gap energy $E_{\mathrm{g}}$, and the wavelength-dependent complex refractive index $n=n+i k$. Throughout this paper we will refer to the real part $n$ as the refractive index and to the imaginary part $k$, to which the absorption is proportional, as the extinction coefficient. $k$ describes the attenuation of electromagnetic waves 

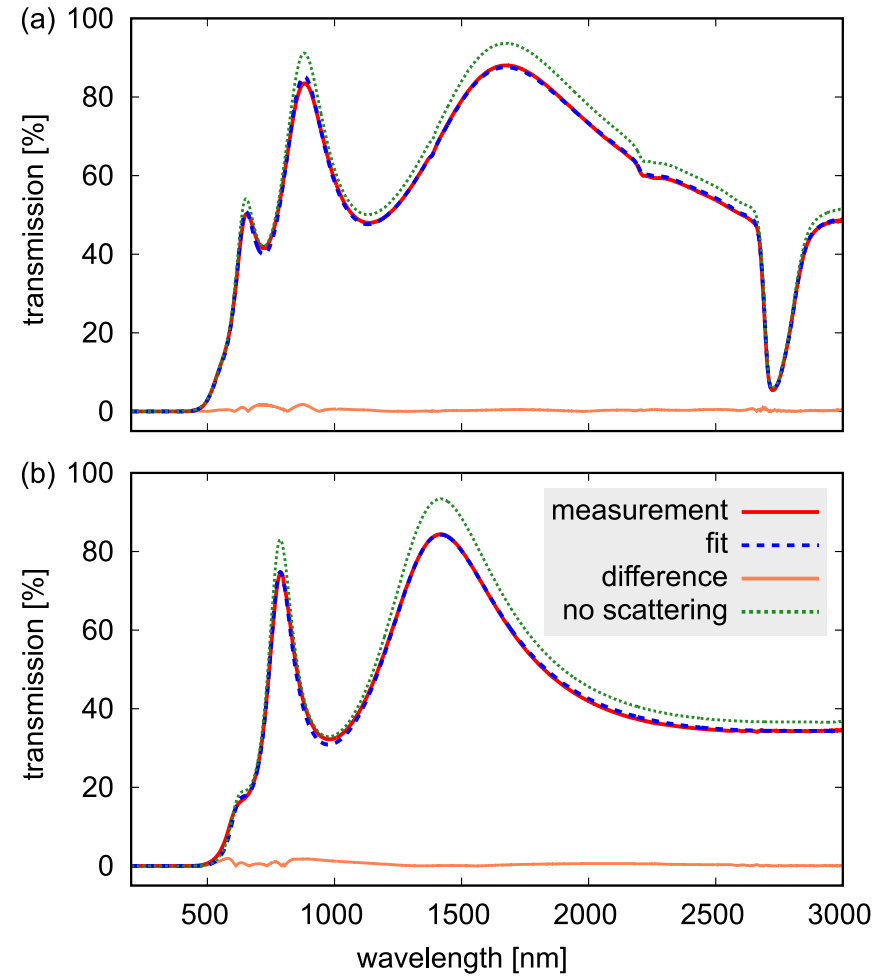

FIG. 1. Examples for transmission spectra (red, solid lines), fits made with SCOUT (blue, dashed lines), and their difference (orange, solid lines). (a) Spectrum of coating F1. The large dip around $2700 \mathrm{~nm}$ and the smaller dips at 2200 and $1400 \mathrm{~nm}$ originate from $\mathrm{OH}$ in the Corning 7980 substrate. (b) Spectrum of coating T3, which was deposited on Corning 7979, which has lower $\mathrm{OH}$ content. The green, dotted lines show the modeled transmission spectra without scattering.

propagating through a medium, i.e., the absorption normalized to a distance. While it is proportional to the absorption, it is thickness independent and can therefore be compared for the different coatings. At the relevant wavelengths, $k$ is too low to be determined accurately from the transmission spectrum and is therefore measured separately (see Sec. II C).

Figure 1 shows examples of measured coating transmission spectra (red lines), fits obtained from the scOUT software (blue, dashed lines), and the difference of both spectra (orange, solid lines). The green dotted line shows the same fit with the scattering term set to zero. In Fig. 1(a), where a Corning 7980 substrate was used, transmission dips (equal to absorption peaks) arising from the $\mathrm{OH}$ content can be seen at about 1400, 2200, and $2700 \mathrm{~nm}$. These absorption peaks are not present in low-OH Corning 7979 substrates [Fig. 1(b)].

\section{Photothermal common-path interferometry}

The optical absorption of the coatings was measured by photothermal common-path interferometry (PCI) [30]. This method uses two laser beams: a high power, small diameter pump beam and a low power, large diameter probe beam. The beams cross at a small angle on the surface of the test substrate. Power from the pump beam absorbed in the coating heats the substrate. This leads to a change of the refractive

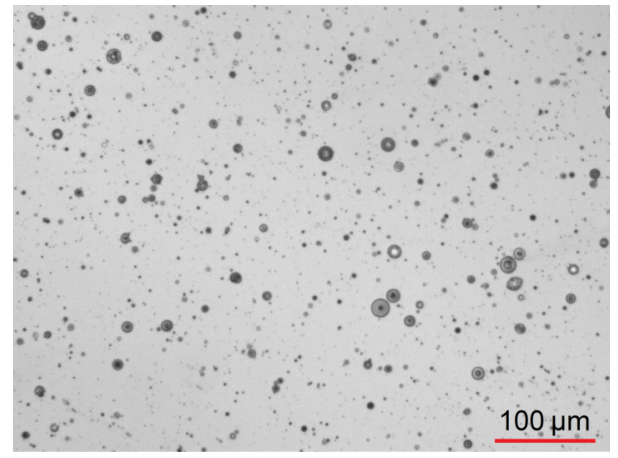

FIG. 2. Example of a microscopic image revealing microcrystals. Shown is an area of $0.60 \times 0.45 \mathrm{~mm}^{2}$ in the center of the coating F1. The images were used to analyze the amount and size of microcrystals on each coating.

index, resulting in the formation of a thermal lens. Part of the probe beam passes through the thermal lens and interferes with the remaining, unaffected part of the probe beam. The interference pattern is imaged onto a photodiode, producing a signal proportional to the absorption. Comparison to the signal from a calibration sample of known absorption allows the coating absorption to be found.

\section{Microcrystal analysis}

A Leica DM4000 M microscope was used to image the samples, revealing microcrystals in every coating (see Fig. 2). This is a transmission microscope and therefore allows all crystals within the volume of a sample to be seen. An image of the center of each sample was recorded. The number of microcrystals in a $0.60 \times 0.45 \mathrm{~mm}^{2}$ area, and the crystals' average diameter, were analyzed using the program IMAGEJ [31]. This information was used to exclude possible correlations between the microcrystals and other parameters investigated.

For the coatings discussed in this paper, no correlations between the number or size of microcrystals and the parameters $k, E_{\mathrm{g}}$, or $n$ were found. However, some further coatings were excluded from our analysis, for which the target showed a deep dent after deposition, caused by the laser scanning not having been well aligned. These coatings showed an unusually high number of crystals and also a high $k$. However, as we did not observe such a correlation for all of the other coatings, we conclude that the high number of crystals is unlikely to be the origin of the high $k$. We assume it to be rather an indicator that other reasons, e.g., a high deposition rate resulting from an irregular material ablation, caused a high $k$. For all the remaining coatings $>85 \%$ of the area was amorphous; for the majority of coatings $>90 \%$ was amorphous.

\section{OPTICAL ABSORPTION}

The silicon targets used for the coatings of all three series were made from the same (undoped, float zone) crystal and the laser alignment on the target was kept as constant as possible. 
TABLE I. List of coatings discussed in this paper and the relevant deposition parameters and measurement results. For some of the coatings an additional comment points out irregularities during the deposition procedure (see Sec. III B). The data of these coatings are marked in green in all plots.

\begin{tabular}{|c|c|c|c|c|c|c|c|c|c|c|c|}
\hline \multirow[b]{2}{*}{ Name } & \multirow[b]{2}{*}{$E(\mathrm{~mJ})$} & \multirow[b]{2}{*}{$f(\mathrm{~Hz})$} & \multirow[b]{2}{*}{$N \times 10^{3}$} & \multirow[b]{2}{*}{$T\left({ }^{\circ} \mathrm{C}\right)$} & \multicolumn{2}{|c|}{$k \times 10^{-3}$} & \multirow[b]{2}{*}{$t(\mathrm{~nm})$} & \multirow[b]{2}{*}{ rate $(\mathrm{pm} / \mathrm{s})$} & \multirow[b]{2}{*}{$E_{\mathrm{g}}(\mathrm{eV})$} & \multirow{2}{*}{$\begin{array}{c}n \\
1550 \mathrm{~nm}^{\mathrm{e}}\end{array}$} & \multirow[b]{2}{*}{ Comment $^{\mathrm{d}}$} \\
\hline & & & & & $1550 \mathrm{~nm}$ & $2000 \mathrm{~nm}$ & & & & & \\
\hline \multicolumn{12}{|c|}{ Frequency series } \\
\hline $\mathrm{F} 1^{\mathrm{a}}$ & 550 & 5 & 150 & $\mathrm{RT}^{\mathrm{c}}$ & 1.51 & & 300 & 10.0 & 1.78 & 2.80 & \\
\hline $\mathrm{F} 2^{\mathrm{a}}$ & 550 & 10 & 150 & RT & 1.32 & & 260 & 17.4 & 1.85 & 2.86 & \\
\hline $\mathrm{F}^{\mathrm{a}}$ & 550 & 20 & 150 & RT & 2.09 & & 248 & 33.1 & 1.83 & 2.95 & \\
\hline $\mathrm{F} 4^{\mathrm{a}}$ & 550 & 30 & 150 & RT & 1.80 & & 101 & 20.4 & 2.44 & 2.68 & High pressure \\
\hline $\mathrm{F}^{\mathrm{a}}$ & 550 & 40 & 150 & RT & 2.52 & & 142 & 37.9 & 1.75 & 3.11 & \\
\hline $\mathrm{F}^{\mathrm{a}}$ & 550 & 50 & 150 & RT & 3.00 & & 94 & 31.4 & 1.87 & 3.10 & Possibly hit clamp \\
\hline \multicolumn{12}{|c|}{ Energy series } \\
\hline $\mathrm{E} 1^{\mathrm{a}}$ & 400 & 10 & 150 & RT & 1.01 & & 285 & 19.0 & 1.80 & 2.85 & \\
\hline $\mathrm{E} 2^{\mathrm{a}}$ & 550 & 10 & 150 & RT & 1.31 & & 400 & 26.6 & 1.79 & 2.89 & \\
\hline $\mathrm{E} 3^{\mathrm{a}}$ & 550 & 10 & 150 & RT & 1.23 & & 353 & 23.5 & 1.82 & 2.79 & \\
\hline $\mathrm{E} 4^{\mathrm{a}}$ & 650 & 10 & 150 & RT & 1.47 & & 298 & 19.9 & 1.83 & 2.90 & \\
\hline $\mathrm{E} 5^{\mathrm{a}}$ & 750 & 10 & 150 & RT & 2.06 & & 499 & 33.3 & 1.80 & 3.04 & \\
\hline $\mathrm{E}^{\mathrm{a}}$ & 850 & 10 & 150 & RT & 2.82 & & 535 & 35.7 & 1.79 & 3.04 & \\
\hline \multicolumn{12}{|c|}{ Temperature series } \\
\hline $\mathrm{T} 1^{\mathrm{b}}$ & 550 & 15 & 200 & RT & 2.06 & 0.60 & 291 & 21.8 & 1.82 & 2.99 & \\
\hline $\mathrm{T} 2^{\mathrm{b}}$ & 550 & 15 & 200 & $100^{\circ} \mathrm{C}$ & 3.13 & 1.02 & 176 & 13.2 & 1.50 & 3.41 & \\
\hline $\mathrm{T} 3^{\mathrm{b}}$ & 550 & 15 & 200 & $200^{\circ} \mathrm{C}$ & 2.39 & 0.85 & 196 & 14.7 & 1.73 & 3.60 & \\
\hline $\mathrm{T} 4^{\mathrm{b}}$ & 550 & 15 & 200 & $300^{\circ} \mathrm{C}$ & 3.10 & 1.23 & 148 & 11.1 & 1.58 & 3.65 & \\
\hline $\mathrm{T} 5^{\mathrm{b}}$ & 550 & 15 & 200 & $400^{\circ} \mathrm{C}$ & 2.18 & 0.61 & 178 & 13.4 & $1.82_{-0.34}^{+0}$ & 3.65 & \\
\hline $\mathrm{T} 6^{\mathrm{b}}$ & 550 & 15 & 200 & $470^{\circ} \mathrm{C}$ & 2.67 & 2.55 & 122 & 9.1 & $1.82_{-0}^{+0.32}$ & 3.57 & Unusual $t$ distr. \\
\hline $\mathrm{T} 7^{\mathrm{b}}$ & 550 & 15 & 200 & $500^{\circ} \mathrm{C}$ & 2.08 & 0.39 & 131 & 9.9 & $2.47^{-0}$ & 3.53 & $\begin{array}{l}\text { silver foil melted, } \\
\text { unusual } t \text { distr. }\end{array}$ \\
\hline
\end{tabular}

${ }^{\mathrm{a} C o r n i n g}$ C7980 substrate.

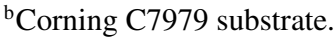

${ }^{\mathrm{c}}$ Room temperature, about $25^{\circ} \mathrm{C}$.

${ }^{\mathrm{d}}$ Data for coatings with comment are marked green in all plots.

${ }^{\mathrm{e}}$ At $2000 \mathrm{~nm}, n$ is slightly lower but within $1 \%$ of the $1550-\mathrm{nm}$ value for all coatings.

In Sec. III A, we present $k$ of all coatings discussed in this paper. Section III B discusses some coatings with unusual features for which the results have to be considered with some care.

\section{A. Absorption as a function of deposition parameters}

In the first series, six coatings were produced using different laser-pulse frequencies between 5 and $50 \mathrm{~Hz}$. The laserpulse energy was $550 \mathrm{~mJ}, N=150000$ laser pulses per coating were used, and the substrate being coated was kept at RT.

The thickness $t$ of the coatings was determined from transmission spectra using SCOUT (see Sec. II B) and used to calculate $k$ from the absorption measured using PCI. Table I shows the deposition parameters and main measurement results for all of the coatings discussed in this paper. It is interesting to note that $t$ decreased as the pulse frequency increased (for $N=$ const).

Figure 3(a) shows $k$ measured at $1550 \mathrm{~nm}$ as a function of laser-pulse frequency. The absorption of each coating was measured multiple times at different positions and the standard deviation arising from averaging of the results was calculated. For each individual absorption measurement an error of approximately $10 \%$ was assumed due to the calibration of the measurement. The error bars represent the $10 \%$ error or the standard deviation for coatings where it exceeded $10 \%$. For all six coatings, $k$ is between $1 \times 10^{-3}$ and $3 \times 10^{-3}$ with an increasing trend towards higher frequencies. This is the same order of magnitude as previously observed for other aSi films which had not been heat treated after deposition [21,22]. For explanation of the points marked in green, see Sec III B.

In the second series, the laser-pulse energy was varied between 400 and $850 \mathrm{~mJ}$ for a constant frequency of $10 \mathrm{~Hz}$. For these coatings, $t$ increased with deposition energy. $k$ as a function of pulse frequency is shown in Fig. 3(b). It has the same magnitude as for the frequency series and shows an increasing trend with pulse energy. Two coatings were made at $550 \mathrm{~mJ}$ to check reproducibility, and showed a difference in $k$ of $\approx 7 \%$.

Figure 3(c) shows $k$ for the third series of coatings in which the temperature of the substrate was varied between RT and about $500{ }^{\circ} \mathrm{C}$. These coatings were also deposited at a laser-pulse energy of $550 \mathrm{~mJ}$, but at a frequency of $15 \mathrm{~Hz}$. A higher number of $N=200000$ pulses per coating was used. On average, $k$ is slightly higher than for the other two series and seems to be rather constant (or oscillating) with no clear increasing or decreasing trend towards higher temperatures. For this series also two points are marked green and are discussed in more detail in Sec. III B. 

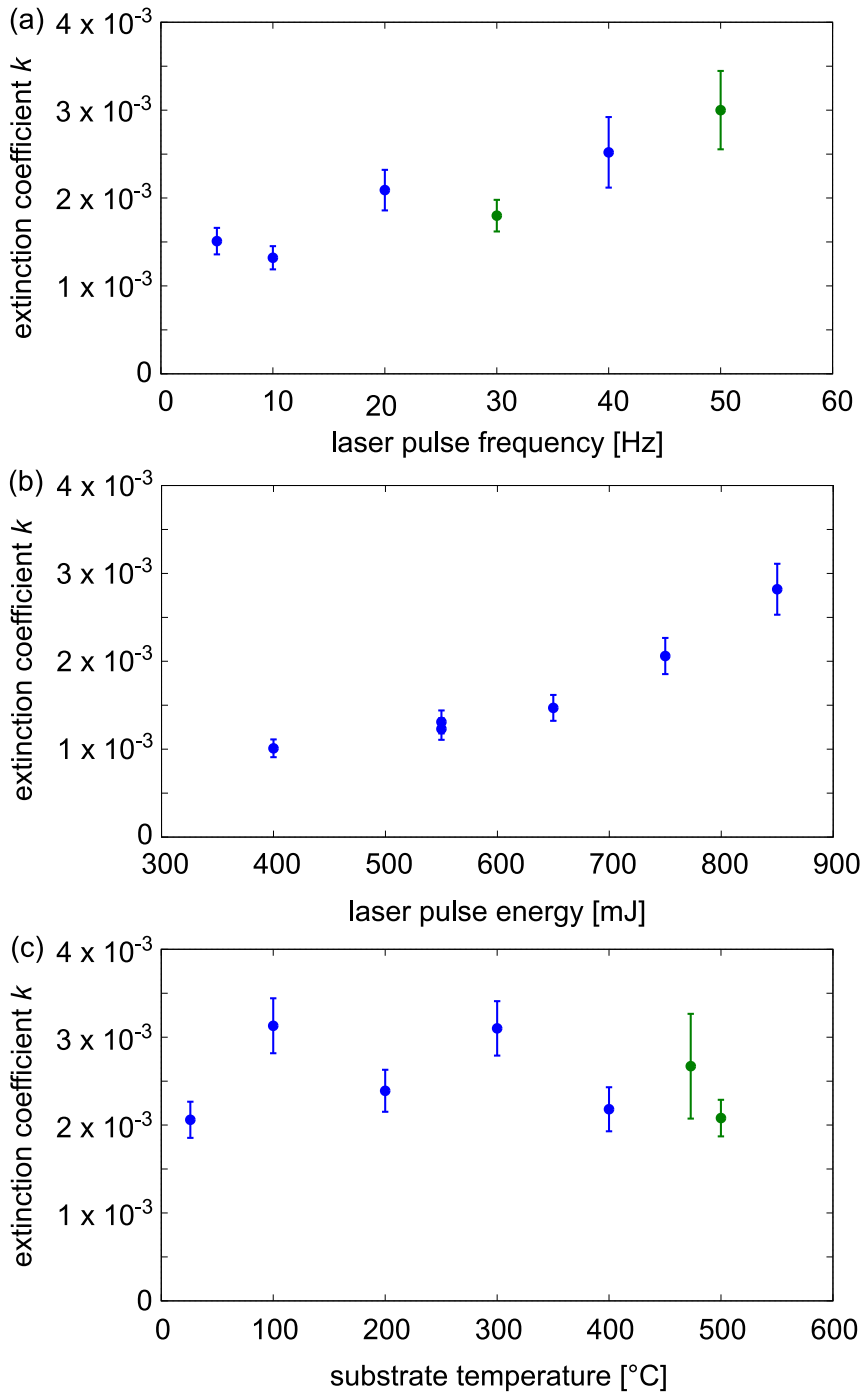

FIG. 3. Extinction coefficient $k$ at $1550 \mathrm{~nm}$ for varied deposition parameters: (a) for a varied laser-pulse frequency (changed time between pulses), (b) for a varied laser-pulse energy, and (c) for a varied temperature of the substrate on which the coating was deposited. The green points represent coatings for which we noticed irregularities during deposition (see Sec. III B).

These results show that varying the deposition parameters can have a significant effect on the absorption of our PLD aSi films. In Sec. IV, we present further investigations into the origins of these absorption changes.

\section{B. Discussion of abnormalities during coating depositions}

A small number of the coating deposition runs had slightly unusual features. While we had no evidence to justify excluding these coatings from our analysis, these points should be considered with care. Therefore they were marked in green in all of the figures throughout this paper.

In the frequency series, during deposition of the $30-\mathrm{Hz}$ coating (F4 in Table I), the pressure in the deposition system increased from $\approx 10^{-5}$ to $\approx 0.4$ mbar, resulting in the coating being thinner than expected. It is unclear if the rising pressure had any effect on other coating parameters, but it represents a potentially significant change in the deposition environment. During the deposition of the coating produced at $50 \mathrm{~Hz}(\mathrm{~F} 6)$, the laser beam possibly hit the clamp holding the target in place. While $k$ of this coating follows the trend, we observed previously that hitting the clamp increases the coating absorption. Therefore this point also has to be considered with care.

In the temperature series, the coatings deposited at about $470{ }^{\circ} \mathrm{C}$ (T6) and $500{ }^{\circ} \mathrm{C}$ (T7) were observed to have a thin area in the middle, surrounded by a thicker ring-shaped area. All other coatings presented in this paper are thickest in the middle, with the thickness decreasing towards the sample edges. The reason for this unusual thickness distribution is unknown. Furthermore, for the $500{ }^{\circ} \mathrm{C}$ point, we observed that some silver foil, used to ensure thermal contact at the back side of the substrate, had been partly melted during deposition. While the absorption of this coating is within the average range, we cannot entirely exclude contamination of the coating, and so we also consider this point with some care.

\section{FURTHER ANALYSIS OF COATING PROPERTIES}

In addition to the correlation of $k$ with the direct deposition parameters, we investigated correlations with the deposition rate and with the mobility gap energy. Furthermore, we investigated how the refractive index of the coatings changed with deposition parameters.

Literature shows that the thicknesses and mobility gaps provided by SCOUT using the OJL model are in good agreement with values obtained by other methods [32-34]. By looking at the goodness of the transmission fits, we also conclude the presented refractive indices to be valid.

\section{A. Deposition rate}

It has been speculated that a low deposition rate may result in low absorption in aSi [21]. In our frequency series, the deposition rate changed directly as more pulses per second mean that more material per second is ablated, so that the coating grows faster. Increasing laser-pulse energy means that each pulse can potentially ablate more material, which would also result in a faster coating growth. Therefore, it was of interest to examine if the similar trends observed for the pulse frequency and pulse energy (see Fig. 3) could be explained by both parameters increasing the deposition rate.

Throughout each coating run, the deposition rate decreased due to the window through which the laser couples into the vacuum system getting coated (unintentionally) with aSi. The coated window absorbs some of the laser power, resulting in less material per laser pulse being ablated from the target. Therefore, here we look at the average deposition rate, which was calculated from the measured film thickness $t$ and from the total deposition time and is given in Table I.

Figure 4(a) shows the measured $k$ at $1550 \mathrm{~nm}$ plotted against the deposition rate with a maximum rate of about $38 \mathrm{pm} / \mathrm{s}$. The deposition rate is found to increase with frequency. This increase is not linear, resulting in a decreasing total coating thickness. Coatings with a higher deposition rate tend to have a higher $k$. The green points (F4 and F6; for more detailed explanation see Table I and Sec. III A) are exceptions to the increasing deposition-rate and absorption 

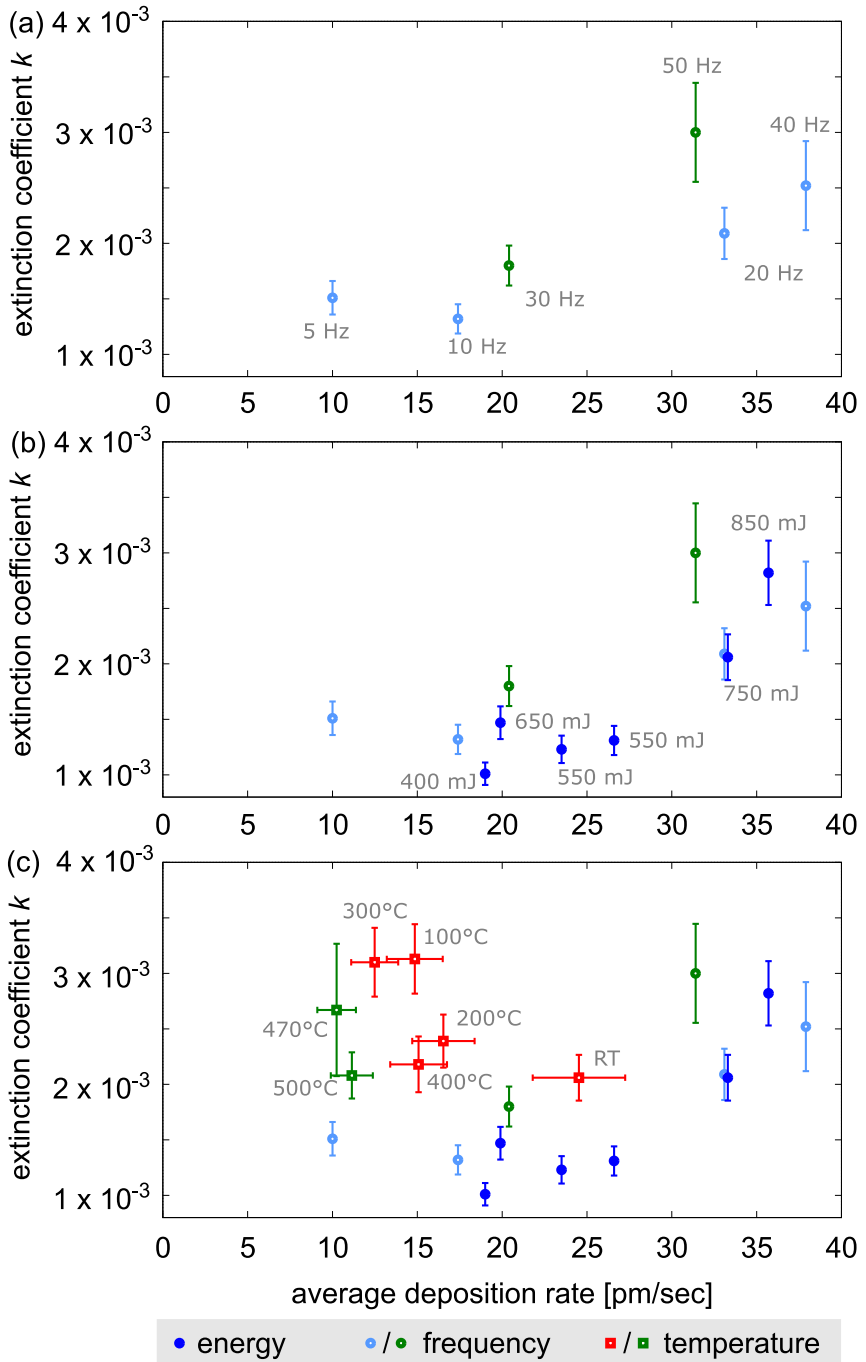

FIG. 4. $k$ at $1550 \mathrm{~nm}$ as a function of average deposition rate. (a) $k$ for a varied laser-pulse repetition frequency. (b) The series with varied laser-pulse energy was added. (c) All three coating series. (For the temperature series a higher number of pulses was used than for the other series, which is indicated by the x-error bars. See main text for more details.) The points are labeled with the relevant deposition parameter to relate points to Fig. 3 (for the green points see Sec. III A).

trends. Without these points, the trend of the remaining points becomes even more clear.

Figure 4(b) shows $k$ at $1550 \mathrm{~nm}$ versus deposition rate for the pulse-energy series, compared to the frequency series. All coatings except the one deposited at $650 \mathrm{~mJ}$ (E4) show an increase in deposition rate with energy and an increase in $k$ with deposition rate. At low deposition rates, $k$ appears to converge to a level of $1-1.5 \times 10^{-3}$. This trend confirms the observations from the frequency series and suggests that the changes in $k$ originate from the changing deposition rate, rather than from any other effect associated with the pulseenergy change.

In Fig. 4(c), the $k$ data of the temperature series are added to the comparison. While for the energy and frequency series $N=150000$ laser pulses per coating were used, $N=200000$ pulses were used for the temperature series. ${ }^{1}$ The temperature series shows an increase in $k$ towards low deposition rateswhen excluding the green points representing coatings deposited at about 470 and $500{ }^{\circ} \mathrm{C}$ (T6 and T7, see Sec. III A) which is the opposite from what we observed on the frequency and energy series. For higher deposition temperatures, the total coating thickness decreased, as can be seen in Table I. However, this overall decrease is not linear but oscillating. The oscillation together with the inverted $k$ trend indicates that changing the substrate temperature has a different effect than changing the frequency or pulse energy. It seems likely that more than one process contributes to changing the coating properties.

It is interesting to note that the temperature series was deposited onto substrates made from Corning 7979 silica rather than the Corning 7980 used for other measurement series. This is discussed further in Sec. V.

\section{B. Refractive index}

From the analysis of the transmission spectra, $n$ as a function of wavelength was obtained. Figure 5(a) shows $n$ as a function of laser-pulse frequency. The blue points show a clear increase in $n$ with increasing frequency. The green points (see Sec. III B) form exceptions to this trend.

Figure 5(b) shows $n$ as a function of laser-pulse energy. For an increase in pulse energy, an increasing trend in $n$ can be observed, at a similar level to that of the frequency series. The point at $10 \mathrm{~Hz}$ in Fig. 5(a) and the $550 \mathrm{~mJ}$ points in Fig. 5(b) had similar deposition parameters resulting in very similar $n$. For both series, $n$ is rather low compared to values for aSi found in literature [35].

Figure 5(c) shows $n$ for the temperature series. While for the coating deposited at RT $n$ is similar to the other two series, a large increase from 3.0 to 3.4 can be observed for deposition at $100{ }^{\circ} \mathrm{C}$. Towards $200^{\circ} \mathrm{C}, n$ further increases, forming a plateau between 200 and $400{ }^{\circ} \mathrm{C}$ at about 3.65. The refractive-index range between 3.4 and 3.65 agrees much better with literature than the lower values observed from the other two series [35]. This seems to be consistent with coating-deposition temperatures of around $100{ }^{\circ} \mathrm{C}$ being quite common.

The two green points (T6 and T7) show a slight decrease in $n$ towards $500{ }^{\circ} \mathrm{C}$. It is unclear if they form exceptions due to the irregularities observed during deposition (see Sec. III B) or if this change is real. It is well known that postdeposition heat treatment improves the optical absorption and also the mechanical loss of aSi up to temperatures between 400 and $500{ }^{\circ} \mathrm{C}$ (varying for coatings made by different vendors and/or with different deposition methods) before the properties get worse again $[19,21,22]$. Therefore, it is possible that the change in refractive index at high temperatures is real, e.g., caused by the coatings starting to crystallize.

\footnotetext{
${ }^{1}$ To make the deposition rate of this series comparable to the other two series, we have added error bars on the deposition rate. The lower end marks the case of a continuous layer growth for $N=200000$ pulses, while the upper end marks the case of no coating growth beyond $N=150000$ pulses.
} 

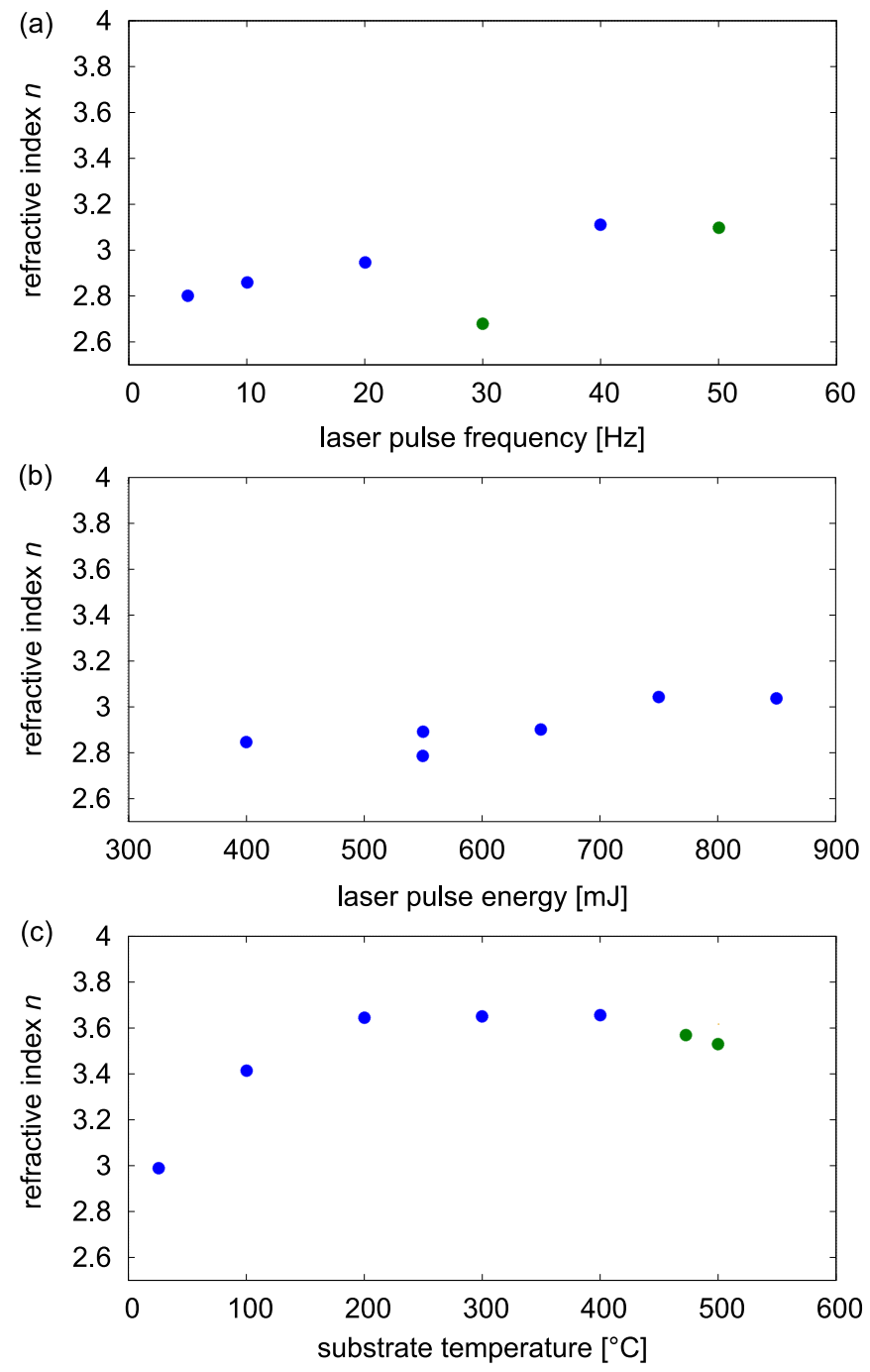

FIG. 5. Refractive index $n$ at $1550 \mathrm{~nm}$ as a function of (a) laserpulse frequency, (b) laser-pulse energy, and (c) substrate temperature during deposition. For better comparability, an identical range was chosen for the $y$ axes. The green points correspond to the green points in Fig. 3 (see Sec. III B for details).

The very smooth trend of the temperature series is interesting as it is the only clear correlation between the deposition temperature and a coating property we observed. This supports the hypothesis that the change in temperature causes two (or more) different processes affecting the coating properties, which interfere and consequently hide some correlations.

\section{Mobility gap energy}

It has been observed previously that aSi films with a higher mobility gap energy $E_{\mathrm{g}}$ tend to have a lower $k$ at $1550 \mathrm{~nm}$ [21]. To investigate this correlation for our coatings, $E_{\mathrm{g}}$ was determined from the transmission spectra using SCOUT (see Table I and Sec. II B).

\section{Mean value and variation of the mobility gap energy}

Figure 6 shows $k$ as a function of the $E_{\mathrm{g}}$ for all three coating series. The energy and frequency series show only a small

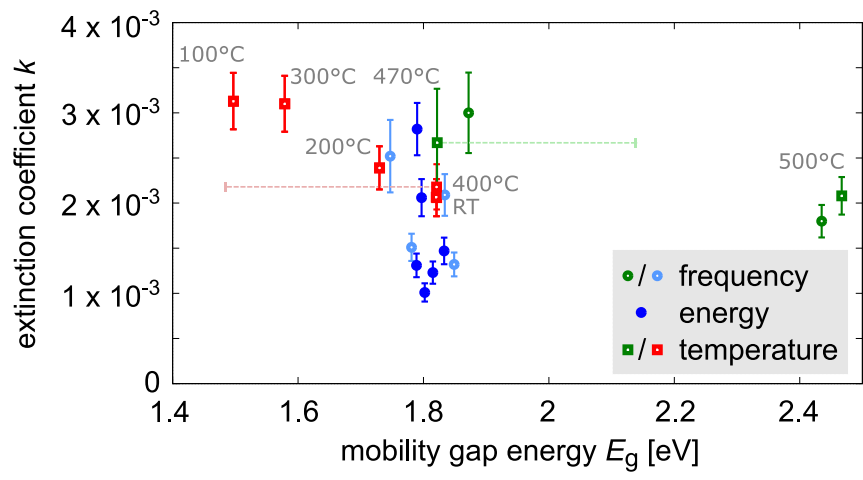

FIG. 6. $k$ as a function of $E_{\mathrm{g}}$ for all three coating series. While for the energy and frequency series $E_{\mathrm{g}}$ groups around $1.8 \mathrm{eV}$, for the temperature series there is more variation, mainly towards lower energies. For two coatings multiple solutions were found indicated by the single-sided error bars (see main text for explanation). For green points, see Sec. III B.

variation in $E_{\mathrm{g}}$, with the exception of one green point ( $\mathrm{F} 4$, see Sec. III B). All the remaining points cluster within $\pm 3 \%$ around the mean value of $1.8 \mathrm{eV}$. This very small change in $E_{\mathrm{g}}$ for a change in $k$ by more than a factor of 3 indicates that the mobility gap energy is unlikely to be the responsible factor for the change in absorption for these two coating series.

The temperature series shows more variation in $E_{\mathrm{g}}$ than the other two series. For two coatings, T5 and T6, the resulting $E_{\mathrm{g}}$ was dependent on the starting parameters of the fit. The points mark the result with the closest agreement between fit and measurement. For T6, a second result for $E_{\mathrm{g}}$ was found located at the end of the dashed, single-sided error bar. For T5, three more results were found, one of which is located at the end of the dashed, single-sided error bar, and two more results are located at 1.60 and $1.61 \mathrm{eV}$.

The coating deposited at a temperature of $500^{\circ} \mathrm{C}$ shows a high mobility gap energy of $E_{\mathrm{g}}=2.5 \mathrm{eV}$. This is the coating deposition run during which the silver foil at the back side of the glass substrate melted, which makes contamination a possibility. A temperature of $500{ }^{\circ} \mathrm{C}$ is also above the temperature at which minimum absorption occurs for some aSi and may lead to significant structural changes. This would be consistent with the decrease in refractive index at the highest temperatures (see Sec. IV B). However, this does not exclude contamination as a possible origin.

When excluding the green points from the temperature series, the mean mobility gap energy is $E_{\mathrm{g}}=1.69 \mathrm{eV}$, varying by $+8 /-11 \%$ for a change in absorption of less than a factor of 2 . The significantly larger variation of $E_{\mathrm{g}}$ in this series compared to the other two series indicates that some effect during deposition of the temperature series affected the bandgap energy - although there is no direct correlation between $E_{\mathrm{g}}$ and $T$ (see Fig. 6).

\section{Correlation of $k$ with the mobility gap energy}

For the temperature series, a significant spread in $E_{\mathrm{g}}$ was observed-with no clear correlation between deposition temperature and $E_{\mathrm{g}}$. This series was deposited on Corning 7979 


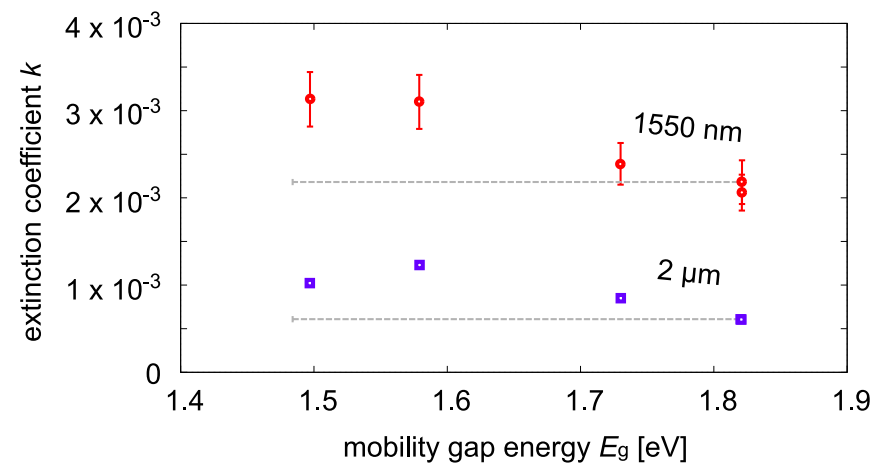

FIG. 7. $k$ at wavelengths of 1550 and $2000 \mathrm{~nm}$ as a function of mobility gap energy for the temperature series. The green points (see Sec. III B) were excluded, making the trend of decreasing $k$ with increasing $E_{\mathrm{g}}$ more clear.

fused silica to allow absorption measurements at a wavelength of $2000 \mathrm{~nm}$ in addition to $1550 \mathrm{~nm}$.

Figure 7 shows $k$ at both wavelengths as a function of $E_{\mathrm{g}}$ for all coatings from the temperature series except coatings T6 and T7 (green points). It is interesting to note that T6 is the only coating for which the absorption at $2000 \mathrm{~nm}$ is not significantly lower than at $1550 \mathrm{~nm}$, while at $1550 \mathrm{~nm}$ it is in the normal range (see Table I). The reason for this is unknown. At both wavelengths, a decreasing trend of $k$ with increasing $E_{\mathrm{g}}$ can be observed. For coating T5, the single-sided error bar indicates multiple fit solutions (see previous section). The solution with the best fit to the measurements follows the decreasing trend of the remaining points. Both effects, the decreasing trend of $k$ with increasing $E_{\mathrm{g}}$ and the lower $k$ at higher wavelengths, confirm the observations made in [21].

\section{DISCUSSION}

Besides a discussion of the absorption correlations presented in the previous sections, here we will discuss a possible effect of the substrate material on the absorption.

\section{A. Absorption processes}

Birney et al. [21] observed a particularly low absorption for aSi deposited by a new technique-which, amongst other unique features, uses a significantly lower deposition rate than used for most commercial coatings. The decrease in $k$ with decreasing deposition rate shown in Figs. 4(a) and 4(b) provides direct evidence for the hypothesis that the low deposition rate is one of the factors responsible for the low absorption.

Furthermore, in [21], two different absorption processes in aSi films are discussed: (1) absorption due to the density of dangling bonds and (2) absorption due to the mobility gap energy of the material. Our frequency and energy series show little change in $E_{\mathrm{g}}$ but significant changes in absorption, as shown in Fig. 6. This suggests that these parameters mainly change the dangling-bond density, which in turn affects $k$.

For the temperature series, a larger spread in mobility gap energy can be observed. We find a decrease in $k$ with increasing mobility gap energy for the majority of these coatings. A similar trend between $k$ and mobility gap energy was observed by [21], although our $k$ values have a higher magnitude.

While $E_{\mathrm{g}}$ somehow seemed to get affected for the temperature series, no clear correlation of $E_{\mathrm{g}}$, or $k$, and deposition temperature could be observed. $k$, as well as the coating thickness and deposition rate, seem to oscillate as the deposition temperature increases. However, the refractive index shows a very smooth trend, increasing up to $200{ }^{\circ} \mathrm{C}$ and then forming a plateau. This indicates that the change in temperature possibly causes different processes affecting the coating properties, which cannot be separated.

\section{B. Influence of the substrate material on the coating properties}

Another interesting observation is that $k$ is rather high for the temperature series compared to the other two series, which can be seen best in Fig. 4. While this could have various reasons, one difference is that the temperature series was deposited on Corning 7979 substrates, while the other coatings were deposited on Corning 7980. The geometry and surface quality of all samples were identical. One of the major differences is the $\mathrm{OH}$ content, which is at least 800 times lower for 7979 compared to Corning 7980 fused silica.

Figure 4 in [21] shows that $k$ correlates with electron-spin density (dangling bonds per volume), with a general trend of decreasing $k$ with decreasing spin density. Their coatings were deposited on two types of substrate-Corning 7979 and JGS-1, with higher $\mathrm{OH}$ content. ${ }^{2}$ Coatings on the two substrate types showed similar trends, but shifted with respect to each other. Coatings on Corning 7979 seemed to have a dangling-bond density that is below the expected values for the observed $k$ (i.e., the trend is shifted left on the graph). Alternatively, it could be said that the high $k$ cannot be explained by the given number of dangling bonds.

Figure 5 in [21] shows a trend of decreasing $k$ with increasing mobility gap energy $E_{\mathrm{g}}$. However, it is notable that $E_{\mathrm{g}}$ was lower for the coatings deposited on Corning 7979, and higher for the coatings deposited on JGS-1. A lower $E_{\mathrm{g}}$ appears to explain the higher than expected absorption for a certain number of dangling bonds. This is in agreement with the data we present in Fig. 6: For the coatings deposited on Corning 7979, on average, $E_{\mathrm{g}}$ is about $0.11 \mathrm{eV}$ lower than for the coatings made on Corning 7980 (green points excluded).

These observations may imply that Corning 7979 causes a slightly different material structure in the coating than Corning 7980 and JGS-1, which results in a higher $k$. Further investigations are required to identify if this is due to the lower $\mathrm{OH}$ concentration, or to some other reason.

\section{CONCLUSION}

We have studied the influence of deposition rate and deposition temperature on the properties of aSi films produced using PLD. When the deposition rate is directly varied by changing the laser-pulse energy or the pulse repetition rate,

\footnotetext{
${ }^{2}$ JGS-1 and Corning 7980 have similar absorption at 1550 and $2000 \mathrm{~nm}$. As the absorption is dominated by $\mathrm{OH}$, this indicates a similar $\mathrm{OH}$ concentration in both these glasses.
} 
we find that lower optical absorption is obtained at lower deposition rates. No significant change in the mobility gap energy was observed when varying the energy or the frequency, suggesting that these aSi coatings all have a similar structure. We conclude, based partly on previous measurements, that the change in absorption with deposition rate is most likely due to dangling bonds, with a higher concentration of dangling bonds arising at higher deposition rates. These systematic studies have verified the hypothesis made by Birney et al. [21] that low deposition rate is an important factor in obtaining low optical absorption in aSi films.

The effect of varying the substrate temperature was more complicated. No clear correlation between temperature, deposition rate, and absorption was found, but a very smooth trend of refractive index with deposition temperature was observed. Coatings produced at different temperatures did show a spread in mobility gap energies, and coatings with lower mobility gap energy had higher absorption. We hypothesise that changing the deposition temperature results in a change in structure of the aSi film, but that more than one process is responsible for the observed changes.

Furthermore, we found evidence that the aSi absorption may be very different when the film is deposited on substrates of differing $\mathrm{OH}$ content. Further work to understand this effect would be of interest to improve the comparability of
aSi coating performance and the interpretation of the results. Also the ability to use coatings on different substrates of varying purity with predictable performance is of interest as the material selection depends on the application, e.g., the laser wavelengths.

\section{ACKNOWLEDGMENTS}

We are grateful for financial support from the Science and Technology Facilities Council (Grant No. ST/N005422/1), the Royal Society (Grant No. RG110331), the University of Glasgow, and the Deutsche Forschungsgemeinschaft (DFG) under Germany's Excellence Strategy (EXC 2121 "Quantum Universe" Grant No. 390833306) and under DFG Project No. STE 2646/1-1. I.W.M. was supported by a Royal Society Research Fellowship for the majority of this project. R.B., M.M.F., and A.M. are grateful for support through the National Science Foundation (Grant No. PHY-1758669). We are grateful to the International Max Planck Partnership for Measurement and Observation at the Quantum Limit for support, and we thank our colleagues in the LIGO Scientific Collaboration and Virgo collaboration and within the Scottish Universities Physics Alliance for their interest in this work. We also thank Maximilian Eckert for his supporting work with SCOUT. This paper has LIGO Document No. LIGO-P2000037.
[1] B. P. Abbott, R. Abbott, T. D. Abbott, M. R. Abernathy, F. Acernese, K. Ackley, C. Adams, T. Adams, P. Addesso, R. X. Adhikari et al. (LIGO Scientific Collaboration and Virgo Collaboration), Observation of Gravitational Waves from A Binary Black Hole Merger, Phys. Rev. Lett. 116, 061102 (2016).

[2] B. P. Abbott, R. Abbott, T. D. Abbott, S. Abraham, F. Acernese, K. Ackley, C. Adams, R. X. Adhikari, V. B. Adya, C. Affeldt et al. (LIGO Scientific Collaboration and Virgo Collaboration), GWTC-1: A Gravitational-Wave Transient Catalog of Compact Binary Mergers Observed by LIGO and Virgo During the First and Second Observing Runs, Phys. Rev. X 9, 031040 (2019).

[3] B. P. Abbott, R. Abbott, T. D. Abbott, F. Acernese, K. Ackley, C. Adams, T. Adams, P. Addesso, R. X. Adhikari, V. B. Adya et al. (LIGO Scientific Collaboration and Virgo Collaboration), GW170817: Observation of Gravitational Waves from A Binary Neutron Star Inspiral, Phys. Rev. Lett. 119, 161101 (2017).

[4] J. Aasi, B. P. Abbott, R. Abbott, T. Abbott, M. R. Abernathy, K. Ackley, C. Adams, T. Adams, P. Addesso, R. X. Adhikari et al. (LIGO Scientific Collaboration), Advanced LIGO, Classical Quantum Gravity 32, 115012 (2015).

[5] B. P. Abbott, R. Abbott, T. D. Abbott, M. R. Abernathy, F. Acernese, K. Ackley, C. Adams, T. Adams, P. Addesso, R. X. Adhikari et al. (LIGO Scientific Collaboration and Virgo Collaboration), GW150914: The Advanced LIGO Detectors in the Era of First Discoveries, Phys. Rev. Lett. 116, 131103 (2016).

[6] T. Accadia, F. Acernese, M. Alshourbagy, P. Amico, F. Antonucci, S. Aoudia, N. Arnaud, C. Arnault, K. G. Arun, P. Astone et al. (Virgo Collaboration), Virgo: A laser interferometer to detect gravitational waves, JINST 7, P03012 (2012).

[7] G. M. Harry, A. M. Gretarsson, P. R. Saulson, S. E. Kittelberger, S. D. Penn, W. J. Startin, S. Rowan, M. M. Fejer, D. R. M.
Crooks, G. Cagnoli, J. Hough, and N. Nakagawa, Thermal noise in interferometric gravitational wave detectors due to dielectric optical coatings, Classical Quantum Gravity 19, 897 (2002).

[8] J. Degallaix, C. Michel, B. Sassolas, A. Allocca, G. Cagnoli, L. Balzarini, V. Dolique, R. Flaminio, D. Forest, M. Granata, B. Lagrange, N. Straniero, J. Teillon, and L. Pinard, Large and extremely low loss: The unique challenges of gravitational wave mirrors, J. Opt. Soc. Am. A 36, C85 (2019).

[9] M. Granata, A. Amato, L. Balzarini, M. Canepa, J. Degallaix, D. Forest, V. Dolique, L. Mereni, C. Michel, L. Pinard, B. Sassolas, J. Teillon, and G. Cagnoli, Amorphous optical coatings of present gravitational-wave interferometers, Classical Quantum Gravity 37, 095004 (2020).

[10] G. M. Harry, M. R. Abernathy, A. E. Becerra-Toledo, H. Armandula, E. Black, K. Dooley, M. Eichenfield, C. Nwabugwu, A. Villar, D. R. M. Crooks, G. Cagnoli, J. Hough, C. R. How, I. MacLaren, P. Murray, S. Reid, S. Rowan, P. H. Sneddon, M. M. Fejer, R. Route, S. D. Penn, P. Ganau, J.-M. Mackowski, C. Michel, L. Pinard, and A. Remillieux, Titaniadoped tantala/silica coatings for gravitational-wave detection, Classical Quantum Gravity 24, 405 (2007).

[11] R. Flaminio, J. Franc, C. Michel, N. Morgado, L. Pinard, and B. Sassolas, A study of coating mechanical and optical losses in view of reducing mirror thermal noise in gravitational wave detectors, Classical Quantum Gravity 27, 084030 (2010).

[12] Y. Aso, Y. Michimura, K. Somiya, M. Ando, O. Miyakawa, T. Sekiguchi, D. Tatsumi, and H. Yamamoto (The KAGRA Collaboration), Interferometer design of the KAGRA gravitational wave detector, Phys. Rev. D 88, 043007 (2013).

[13] M. Abernathy, F. Acernese, P. Ajith, B. Allen, P. AmaroSeoane, N. Andersson, S. Aoudia, P. Astone, B. Krishnan, L. 
Barack et al., Einstein Telescope (ET) conceptual design study (2011), http://www.et-gw.eu/index.php/etdsdocument.

[14] I. W. Martin, R. Bassiri, R. Nawrodt, M. M. Fejer, A. Gretarsson, E. Gustafson, G. Harry, J. Hough, I. MacLaren, S. Penn, S. Reid, R. Route, S. Rowan, C. Schwarz, P. Seidel, J. Scott, and A. L. Woodcraft, Effect of heat treatment on mechanical dissipation in $\mathrm{Ta}_{2} \mathrm{O}_{5}$ coatings, Classical Quantum Gravity 27, 225020 (2010).

[15] I. W. Martin, R. Nawrodt, K. Craig, C. Schwarz, R. Bassiri, G. Harry, J. Hough, S. Penn, S. Reid, R. Robie, and S. Rowan, Low temperature mechanical dissipation of an ion-beam sputtered silica film, Classical Quantum Gravity 31, 035019 (2014).

[16] M. Granata, K. Craig, G. Cagnoli, C. Carcy, W. Cunningham, J. Degallaix, R. Flaminio, D. Forest, M. Hart, J. Hennig et al., Cryogenic measurements of mechanical loss of high-reflectivity coating and estimation of thermal noise, Opt. Lett. 38, 5268 (2013).

[17] P. G. Murray, I. W. Martin, K. Craig, J. Hough, R. Robie, S. Rowan, M. R. Abernathy, T. Pershing, and S. Penn, Ionbeam sputtered amorphous silicon films for cryogenic precision measurement systems, Phys. Rev. D 92, 062001 (2015).

[18] X. Liu, B. E. White, Jr., R. O. Pohl, E. Iwanizcko, K. M. Jones, A. H. Mahan, B. N. Nelson, R. S. Crandall, and S. Veprek, Amorphous Solid without Low Energy Excitations, Phys. Rev. Lett. 78, 4418 (1997).

[19] J. Steinlechner, I. W. Martin, R. Bassiri, A. Bell, M. M. Fejer, J. Hough, A. Markosyan, R. K. Route, S. Rowan, and Z. Tornasi, Optical absorption of ion-beam sputtered amorphous silicon coatings, Phys. Rev. D 93, 062005 (2016).

[20] M. A. Green and M. J. Keevers, Optical properties of intrinsic silicon at $300 \mathrm{~K}$, Prog. Photovoltaics Res. Appl. 3, 189 (1995).

[21] R. Birney, J. Steinlechner, Z. Tornasi, S. MacFoy, D. Vine, A. S. Bell, D. Gibson, J. Hough, S. Rowan, P. Sortais, S. Sproules, S. Tait, I. W. Martin, and S. Reid, Amorphous Silicon with Extremely Low Absorption: Beating Thermal Noise in Gravitational Astronomy, Phys. Rev. Lett. 121, 191101 (2018).

[22] J. Steinlechner, I. W. Martin, A. S. Bell, J. Hough, M. Fletcher, P. G. Murray, R. Robie, S. Rowan, and R. Schnabel, Silicon-
Based Optical Mirror Coatings for Ultrahigh Precision Metrology and Sensing, Phys. Rev. Lett. 120, 263602 (2018).

[23] P. J. Zanzucchi, C. R. Wronski, and D. E. Carlson, Optical and photoconductive properties of discharge-produced amorphous silicon, J. Appl. Phys. 48, 5227 (1977).

[24] Heitronics Infrarot Messtechnik GmbH, KT19.82 II, www. heitronics.com.

[25] F. Tellkamp, PLD-Grown Thin Film Saturable Absorbers, Ph.D. thesis, Universität Hamburg, 2012.

[26] Agilent, Cary 5000 UV-Vis-NIR, www.agilent.com.

[27] W. Theiss, Hard- and Software, www.wtheiss.com.

[28] S. K. O'Leary, S. R. Johnson, and P. K. Lim, The relationship between the distribution of electronic states and the optical absorption spectrum of an amorphous semiconductor: An empirical analysis, J. Appl. Phys. 82, 3334 (1997).

[29] V. Lucarini, J. J. Saarinen, K.-E. Peiponen, and E. M. Vartiainen, Kramers-Kronig Relations in Optical Materials Research, Springer Series in Optical Sciences, Vol. 110 (Springer, New York, 2005).

[30] A. Alexandrovski, M. Fejer, A. Markosian, and R. Route, Photothermal common-path interferometry (PCI): New developments, in Solid State Lasers XVIII: Technology and Devices, Vol. 7193, edited by W. Andrew Clarkson, Norman Hodgson, and Ramesh K. Shori, International Society for Optics and Photonics, Vol. 7193 (SPIE, San Jose, California, 2009), pp. 79-91, https://doi.org/10.1117/12.814813.

[31] National Institute of Health, https://imagej.nih.gov/ij/.

[32] N. Musila, M. Munji, J. Simiyu, E. Masika, and R. Nyenge, Optical properties and analysis of OJL model's electronic interband transition parameters of $\mathrm{TiO}_{2}$ films, Path Science 4, 3001 (2018).

[33] A. Solieman and A. A. Abu-Sehly, Modelling of optical properties of amorphous selenium thin films, Physica B: Condensed Matter 405, 1101 (2010).

[34] A. A. El-Amin and A. Solieman, Influence of heat treatment on the optical properties of thermal evaporated $\mathrm{SnO}_{2}$ thin films, Silicon 8, 517 (2016).

[35] D. T. Pierce and W. E. Spicer, Electronic structure of amorphous Si from photoemission and optical studies, Phys. Rev. B 5, 3017 (1972). 